\title{
High-Resolution Spectroscopy of Bonding in a Novel $\mathrm{BeP}_{2} \mathrm{~N}_{4}$ Compound
}

\author{
Teresa Dennenwaldt, ${ }^{1}$ Jim Ciston, ${ }^{2}$ Ulrich Dahmen, ${ }^{2}$ Wai-Yim Ching, ${ }^{3}$ Florian J. Pucher, ${ }^{1}$ \\ Wolfgang Schnick, ${ }^{1}$ and Christina Scheu ${ }^{1, *}$ \\ ${ }^{1}$ Department of Chemistry and Center for NanoScience, Ludwig-Maximilians-Universität München, Butenandtstr. 5-13, 81377 \\ Munich, Germany \\ ${ }^{2}$ Lawrence Berkeley National Laboratory, National Center for Electron Microscopy, Berkeley, CA 94720, USA \\ ${ }^{3}$ Department of Physics and Astronomy, University of Missouri-Kansas City, Kansas City, MO 64110, USA
}

\begin{abstract}
The recently discovered compound $\mathrm{BeP}_{2} \mathrm{~N}_{4}$ that crystallizes in the phenakite-type structure has potential application as a high strength optoelectronic material. Therefore, it is important to analyze experimentally the electronic structure, which was done in the present work by monochromated electron energy-loss spectroscopy. The detection of Be is challenging due to its low atomic number and easy removal under electron bombardment. We were able to determine the bonding behavior and coordination of the individual atomic species including Be. This is evident from a good agreement between experimental electron energy-loss near-edge structures of the Be-K-, $\mathrm{P}_{-} \mathrm{L}_{2,3^{-}}$, and $\mathrm{N}-\mathrm{K}$-edges and density functional theory calculations.
\end{abstract}

Key words: EELS, TEM, DFT, inorganic compounds/condensed matter

\section{INTRODUCTION}

Nitride materials are known for their high strength and stability at elevated temperatures and mechanical stress because of their rather covalent chemical bonds to nitrogen (de la Cruz et al., 2004). In addition, they offer many possible applications in optoelectronics due to their wide-band gap semiconductor character. For example, $\mathrm{BeCN}_{2}$ (Lambrecht \& Segall, 1992) and $\mathrm{Be}_{3} \mathrm{~N}_{2}$ (Armenta \& Reyes-Serrato, 2001) possess direct band gaps between 4 and $6 \mathrm{eV}$. Nitrogen forms short bonds in these compounds leading to comparable properties with other wide-band gap semiconductors such as diamond (Mokhtari \& Akbarzadeh, 2003). $\mathrm{Be}_{3} \mathrm{~N}_{2}$ exhibits mechanical behavior similar to $\mathrm{SiC}$ and $\mathrm{Si}_{3} \mathrm{~N}_{4}$ (Armenta \& Reyes-Serrato, 2001). Another structurally related nonmetal nitride is $\mathrm{P}_{3} \mathrm{~N}_{5}$, which has been fully characterized (Schnick et al., 1996; Horstmann et al., 1997). Density functional theory (DFT) calculations on this material predict outstanding mechanical properties comparable to $\mathrm{BN}$ and $\mathrm{Si}_{3} \mathrm{~N}_{4}$ (Kroll \& Schnick, 2002). $\alpha-\mathrm{P}_{3} \mathrm{~N}_{5}$ can be described as a covalent network of $\mathrm{PN}_{4}$ tetrahedra sharing edges and corners (Horstmann et al., 1997). Starting from the compounds $\mathrm{P}_{3} \mathrm{~N}_{5}$ and $\mathrm{Be}_{3} \mathrm{~N}_{2}$ a new hard material $\mathrm{BeP}_{2} \mathrm{~N}_{4}$ has been synthesized recently via the multi-anvil high-pressure/high-temperature method (Pucher et al., 2010). This true double nitride, which crystallizes in the phenakite-type structure, is isoelectronic and isostructural to $\mathrm{Be}_{2} \mathrm{SiO}_{4}$ and $\beta-\mathrm{Si}_{3} \mathrm{~N}_{4}$. Like $\alpha-\mathrm{P}_{3} \mathrm{~N}_{5}$ and $\alpha-\mathrm{Be}_{3} \mathrm{~N}_{2}$, $\mathrm{BeP}_{2} \mathrm{~N}_{4}$ is made of $\mathrm{TN}_{4}$ tetrahedra $(\mathrm{T}=\mathrm{Be}, \mathrm{P})$. Under high pressure, $\beta-\mathrm{Si}_{3} \mathrm{~N}_{4}$ transforms into the spinel-type $\gamma-\mathrm{Si}_{3} \mathrm{~N}_{4}$, which is known as a very hard material (Zerr et al., 1999).

Received July 29, 2013; accepted December 4, 2013

*Corresponding author. Christina.Scheu@cup.uni-muenchen.de
By analogy, $\mathrm{BeP}_{2} \mathrm{~N}_{4}$ is expected to transform under high pressure into the spinel structure as well, leading to another super hard material where the coordination number of $\mathrm{P}$ would increase from 4 to 6 (Pucher et al., 2010; Ching et al., 2011). A sixfold coordination of $P$ in polymeric nitrides has only been predicted for hypothetical $\delta$ - $\mathrm{P}_{3} \mathrm{~N}_{5}$ but could not be observed in any known material (Kroll \& Schnick, 2002). The synthesis of spinel-type $\mathrm{BeP}_{2} \mathrm{~N}_{4}$ is challenging and currently under experimental investigation.

The phenakite-type structure of $\mathrm{BeP}_{2} \mathrm{~N}_{4}$ is primitive rhombohedral with lattice parameters of $a=12.6897 \AA$ and $c=8.3469 \AA$ (space group $R \overline{3}$, no. $148, Z=18$; Pucher et al., 2010). Both Be and $\mathrm{P}$ are tetrahedrally coordinated by four $\mathrm{N}$ atoms resulting in corner sharing dreier, vierer, and sechser rings of $\mathrm{BeN}_{4}$ and $\mathrm{PN}_{4}$ tetrahedra. The terms dreier, vierer, and sechser ring were introduced by Liebau (1985). A sechser ring consists of six tetrahedra ( $\mathrm{P}$ and $\mathrm{Be}$ as central atoms) and analogously this is valid for dreier (three tetrahedra) and vierer rings (four tetrahedra). The $\mathrm{N}$ atoms are arranged in a trigonal planar coordination by one Be and two $\mathrm{P}$ atoms (Pucher et al., 2010). There are two crystallographically unique $P$ and four unique $\mathrm{N}$ atoms, which differ in their bond lengths and angles to the respective neighboring atoms. N1 and N2 are coordinated to P1 and P2 as well as to Be. N3 does not coordinate to P1 but twice to P2 and vice versa for N4. Figure 1a shows the crystal structure with [001] viewing direction. Diverse physical features such as the electronic, spectroscopic, and mechanical properties of $\mathrm{BeP}_{2} \mathrm{~N}_{4}$ were recently investigated using DFT calculations (Ching et al., 2011). In general, DFT methods have been successfully used for electronic structure and property calculations of various compounds (Nelhiebel et al., 1999; Lie et al., 2000). The results of Ching et al. (2011) indicate that the phenakite- as well as the spinel-type phases are wide-band gap 
semiconductors leading to promising applications, e.g. in optoelectronics. The orthogonalized linear combination of atomic orbitals (OLCAO), which is based on the local density approximation of DFT, has been used as the main method for predicting the electronic structures and properties of the phenakite- and the spinel-type polymorph of $\mathrm{BeP}_{2} \mathrm{~N}_{4}$ (Ching, 1990; Ching \& Rulis, 2012).

Electron energy-loss spectroscopy (EELS) is a widely used method in transmission electron microscopy (TEM) to analyze the chemical and electronic structure of materials, locally and in detail (Brydson, 2001; Egerton, 2011). One limiting factor for the energy resolution of this technique is the initial energy spread of the electron source. The energy resolution can be enhanced by using a monochromator resulting in values of around $0.1 \mathrm{eV}$ (Kahl \& Rose, 2000; Uhlemann \& Haider, 2002). With this energy resolution it is possible to determine details in the electron energy-loss near-edge structures (ELNES) related to the electronic structure and bonding effects, as well as to investigate the low-loss region in depth to determine, e.g. the dielectric function or band gaps of materials on a local scale (Lazar et al., 2003; Erni \& Browning, 2005; Gu et al., 2007; Kuykendall et al., 2007; Schaffer et al., 2010). The electronic and structural properties of several nitrides have been studied successfully with EELS (de la Cruz et al., 2004; Ziegler et al., 2004) and it has been shown that the ELNES is strongly dependent on the bond lengths and angles. In this paper we present EELS data acquired with a monochromator from phenakite-type crystals of $\mathrm{BeP}_{2} \mathrm{~N}_{4}$. The experimental ELNES of the Be-K-, $\mathrm{P}-\mathrm{L}_{2,3^{-}}$, and the N-K-edges are interpreted with the help of the calculated data published recently (Ching et al., 2011). For the calculation of the ELNES the supercell-OLCAO (Mo \& Ching, 2000; Ching \& Rulis, 2009) method was applied. In this technique the core-hole effect and the dipole matrix elements calculated from $a b$ initio wave functions are included. To adjust the calculated spectra to experimental conditions the calculated ELNES were broadened by a Gaussian with a fullwidth at half-maximum (FWHM) of $1.0 \mathrm{eV}$. As will be shown in the Results and Discussion section, this value describes nicely the damping occurring in the experiments due to broadening caused by life time of the excited state and corehole. As the material contains small amounts of impurities and cannot be synthesized in large quantities, EELS in a TEM is required for analyzing the local electronic structure of these phenakite-type crystals.

\section{Methods And Materials}

Monochromated EELS data were taken on the NCEM TEAM 0.5 microscope at $80 \mathrm{kV}$ in Berkeley. This doubleaberration corrected (scanning) TEM (STEM/TEM) is based on a FEI Titan 80-300 kV. It is equipped with a special high-brightness Schottky field emission electron source, a gun monochromator, two CEOS hexapole-type spherical aberration correctors (Haider et al., 2008), and a Gatan high-resolution GIF Tridiem energy filter. The FWHM of the zero-loss peak (ZLP) was around $0.12 \mathrm{eV}$ during our measurements done in diffraction mode. The ELNES data were taken with a dispersion of $0.05 \mathrm{eV} /$ channel leading to a high-energy resolution and a good signal-to-noise ratio. We used a $1 \mathrm{~mm}$ entrance aperture for the spectrometer and a camera length of $43 \mathrm{~mm}$ resulting in a collection angle of $12.3 \mathrm{mrad}$. The investigated sample was thin enough (smaller than 0.3 times the inelastic mean free path), so no deconvolution of our data was necessary. All EEL spectra were corrected for channel-to-channel gain variation and dark current. The background was subtracted using a power-law fitting procedure (Egerton, 2011). High-resolution TEM (HRTEM) studies were performed in Munich on a Titan $80-300 \mathrm{keV}$ $\mathrm{S} / \mathrm{TEM}$ equipped with an energy-dispersive $\mathrm{X}$-ray detector and a Gatan energy filter for EELS measurements as well as in Berkeley on a Titan 80-300 keV S/TEM. HRTEM image simulation was conducted using the program MacTempasX.

The $\mathrm{BeP}_{2} \mathrm{~N}_{4}$ material was synthesized from $\mathrm{Be}_{3} \mathrm{~N}_{2}$ and $\mathrm{P}_{3} \mathrm{~N}_{5}$ in a Walker-type multi-anvil apparatus at a pressure of $5 \mathrm{GPa}$ and $1,500^{\circ} \mathrm{C}$, as described in the literature (Pucher et al., 2010). The crude product was heated to $680^{\circ} \mathrm{C}$ to remove traces of black phosphorus and to improve the crystallinity of the sample. For TEM investigations the material was pestled, suspended in ethanol, and dropped on a copper grid coated with a lacey carbon film.

\section{Results And Discussion}

Electron diffraction experiments confirmed that the compound is crystalline exhibiting the phenakite-type structure. A HRTEM image and its corresponding diffraction pattern taken in [210] direction are given in Figure 1b. Owing to the high hardness of the material the thickness within the displayed area is increasing, which is visible in the changes of the HRTEM pattern. Nevertheless, a simulated image is shown as an inset in Figure $1 \mathrm{~b}$ in an area of constant thickness. HRTEM image simulations were performed using MacTempasX according to the following parameters: an acceleration voltage of $80 \mathrm{kV}$, a spherical aberration of $C_{s}=-15 \mu \mathrm{m}$, an information limit of $1 \AA$, a defocus spread of $1.2 \mathrm{~nm}$, fifth-order spherical aberration C5 $=3 \mathrm{~mm}$, and a beam convergence of $0.15 \mathrm{mrad}$. The good agreement between experiment and simulation confirms the crystal structure viewed along [210] with the best match for a defocus of $60 \AA$ and a thickness of $44 \AA$ for this investigated area. A sketch is shown in Figure 1c.

Light elements like Be can be knocked out or displaced quickly by the electron beam. This was also detectable in HRTEM studies conducted at $300 \mathrm{keV}$ (Fig. 2). Initially, a thin $\sim 1 \mathrm{~nm}$ amorphous layer is visible at the sample edge, which is because of the mechanical treatment during sample preparation (Fig. 2a). After $1 \mathrm{~min}$, illuminating an area of $\sim 50 \mathrm{~nm}$ in diameter, noticeable changes occur indicating severe damage (mostly knock-on damage) under the chosen conditions (Fig. 2b). In further experiments using similar conditions but operating the microscope at $80 \mathrm{keV}$ revealed a much higher stability even up to $1,000 \mathrm{~s}$ (Figs. 2c, 2d). Nevertheless, to make sure that beam damage effects did not strongly influence the Be-K ELNES, this data was taken with 
a

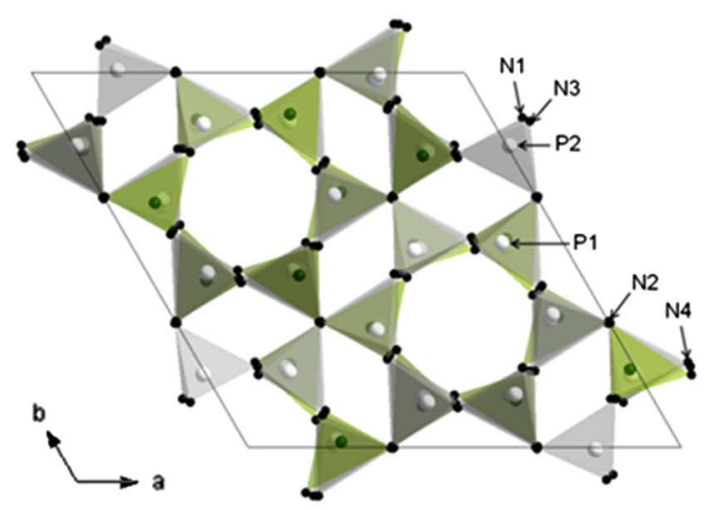

b

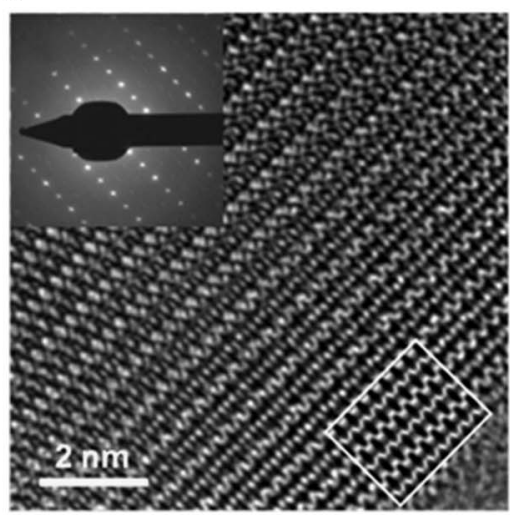

C

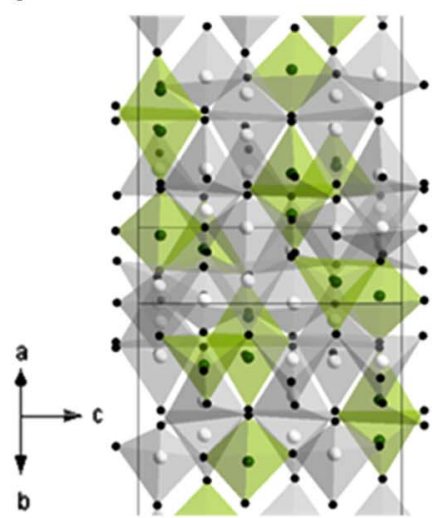

Figure 1. Crystal structure of the phenakite-type phase with $\mathrm{BeN}_{4}$ tetrahedra highlighted in green, and $\mathrm{PN}_{4}$ tetrahedra in gray. (a) Structure viewed along [001]; the different $\mathrm{P}$ and $\mathrm{N}$ sites are indicated; (b) high-resolution transmission electron microscopic image of the phenakite-type polymorph of $\mathrm{BeP}_{2} \mathrm{~N}_{4}$ seen along the [210] direction with an enclosed simulated image and the corresponding diffraction pattern; (c) atomic arrangement of the phenakite-type phase viewed along [210].

shorter acquisition times $(<2 s)$ than the other edges (acquisition times up to 20 s), which is why the experimental spectra of the Be-edge appear noisier.

The experimental as well as the calculated EEL spectra for the phenakite phase are given in Figure 3, where the theoretical spectra are shown in black and the monochromated data in blue. In order to prevent orientation and channeling effects the sample was tilted out of zone axis for EELS data collection. Figure 3 a shows the Be-K-edge with the characteristic features labeled A-D. In the experimental data of the Be-K-edge the first maximum, labeled $\mathrm{A}$, shows the highest intensity and occurs at $116.9 \pm 0.1 \mathrm{eV}$. The scatter in the peak position is estimated by averaging over several independent measurements. In the calculated spectrum this peak appears at $118 \mathrm{eV}$ (Ching et al., 2011). For better comparison of all following features (B-D) we shifted the calculated data by $1.1 \mathrm{eV}$ to lower energy losses (to align the first maximum of the experimental and simulated data) since we are most interested in relative peak distances. A difference in the edge onsets of the calculated and experimental values of $\sim 1 \%$ is commonly reported in the literature (Ching \& Rulis, 2009; Tanaka \& Mizoguchi, 2009). Possible explanations are errors in the calibration of the spectrometer as well as difficulties in calculating excited states. Overall, the general shapes of the experimental and calculated Be-K ELNES are in good agreement. The relative peak distances in the experimental data are $2.5 \mathrm{eV}$ for features $\mathrm{A}$ to $\mathrm{B}, 5.4 \mathrm{eV}$ for features $\mathrm{A}$ to $\mathrm{C}$ and $11.5 \mathrm{eV}$ for A to D. As shown in Table 1, these observed peak distances are in good agreement with the calculated ELNES data. Feature $\mathrm{C}$ is different in the experimental and calculated data, which predicts two features. In a simplified picture, the peak splitting of feature C observed in the calculated ELNES of the Be-K-edge could be attributed to different maxima in the density of hybridized Be $2 p / \mathrm{N} 2 p$ states related to the different $\mathrm{Be}-\mathrm{N}$ bond length. The presence of only one peak $\mathrm{C}$ in the experimental data thus indicates that the predicted atomic arrangement is not fully obtained in the experiment.
It is worth noting that the use of partial density of states has some difficulties and might not allow explaining the origin of the different peaks in general. This is related to the fact that the exact determination of hybridization states is not straightforward and often not advisable, in particular, for complex material systems. The unoccupied conduction band (CB) states are highly delocalized and possess all kinds of mixtures. In addition, the peak structures reflect the strength of the dipole matrix elements included in the calculation for various transitions at different excitation energies in the presence of a core-hole. Thus, it is impossible to know the exact origin of each peak, large and small.

In general, a perfect quantitative agreement between theory and experiment for more complex crystals (such as in the present case) is nearly impossible because of limitations in both theory and in experiments/samples. Many factors such as the core-hole effect, the anisotropy of the material, and possible defects and weighting of the different $\mathrm{P}$ and $\mathrm{N}$ sites must be taken into account. Furthermore, an energydependent damping by a flexible Gaussian is not included in the code to intentionally avoid using different broadening effects at different energy ranges for the sole purpose of having a better agreement with the experiment. Further reasons for the discrepancy between the experimental and calculated ELNES data are the limited accuracy in the calculation related to finite basis expansion, the neglecting of many body effects, and that the synthesized phenakite crystals are not fully relaxed and the equilibrium atomic positions not yet achieved.

Figure $3 \mathrm{~b}$ shows the $\mathrm{P}-\mathrm{L}_{2,3}$-edge, which exhibits more fine structure than the Be-K-edge. The first maximum (A) occurring at $136.1 \pm 0.7 \mathrm{eV}$ in the experimental data is also the highest intensity peak in the spectrum. The calculated first maximum is located at $138.2 \mathrm{eV}$ (Ching et al., 2011) so the calculated data was shifted by $2.1 \mathrm{eV}$ to lower energy losses. Similar to the discussion of Be-K ELNES, the relative peak separations of the minor features (B-E) of the 
a

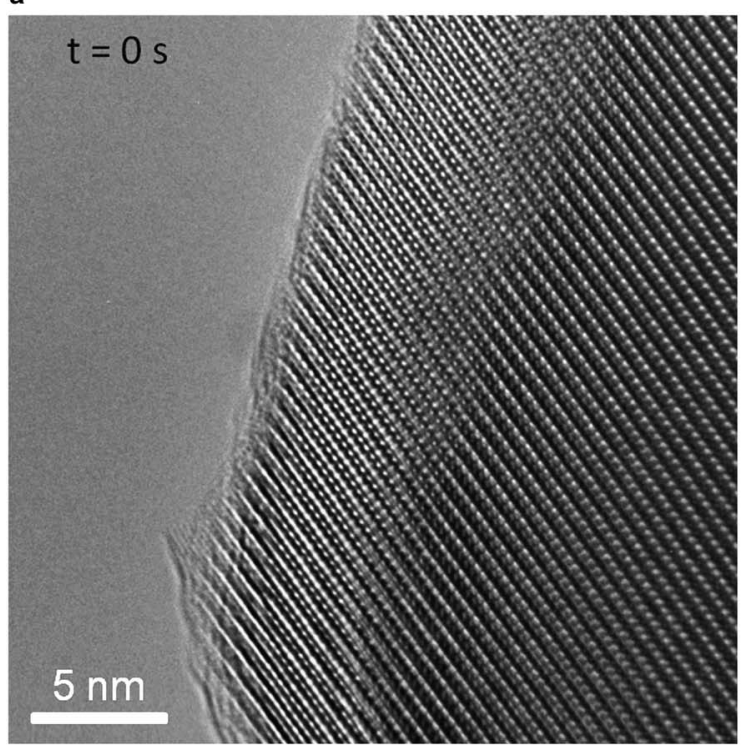

C

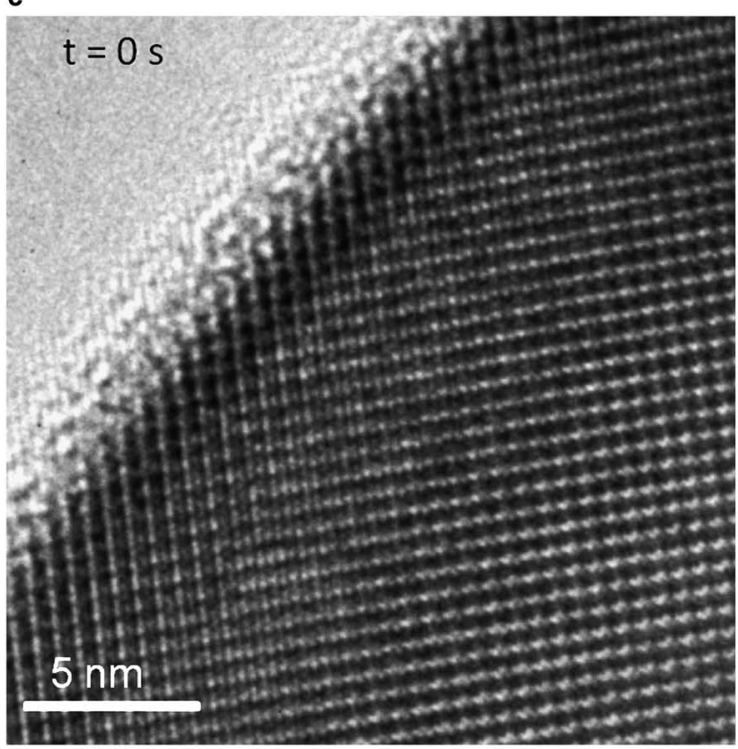

b

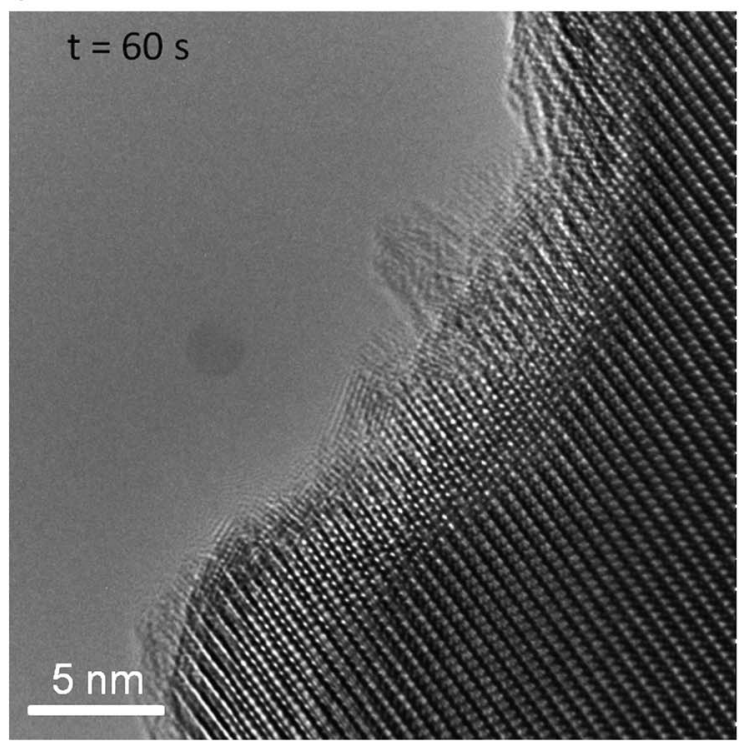

d

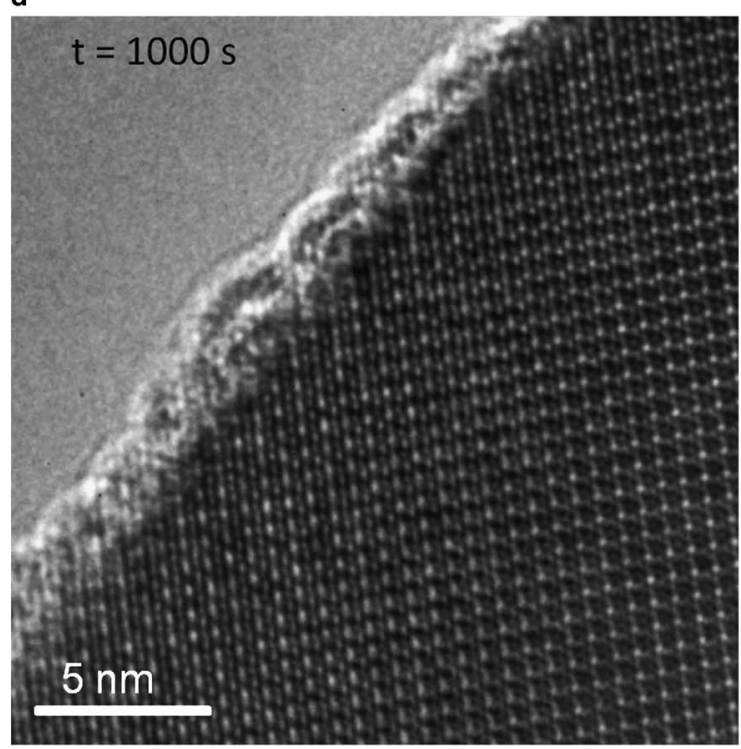

Figure 2. Beam damage study of the phenakite-type $\mathrm{BeP}_{2} \mathrm{~N}_{4}$ phase, in (a) a thin amorphous layer $(\sim 1 \mathrm{~nm})$ is visible at nominal $0 \mathrm{~s}$ irradiation time at $300 \mathrm{kV},(\mathbf{b})$ exhibits a severe damage after illuminating the sample for $60 \mathrm{~s}$ operating the transmission electron microscope at $300 \mathrm{kV}$. In (c) a comparable sample area with a thin amorphous layer is shown at nominal $0 \mathrm{~s}$ irradiation time at $80 \mathrm{kV}$ and (d) shows no considerable damage even after illuminating the area for 1,000 s.

$\mathrm{P}-\mathrm{L}_{2,3}$-edge again show good agreement with the calculated data. Features A, B, and C are present in the calculated data set as well as in the experimental measurement. The weak shoulder on the high-energy side of peak $\mathrm{A}$ is not visible in the calculated data. This small shoulder is the signature of $\mathrm{L}_{2}, \mathrm{~L}_{3}$ splitting $(\sim 1 \mathrm{eV})$, which is resolved by the monochromated data. The calculated L-edge is only for $\mathrm{L}_{3}$, and omits any contributions from $\mathrm{L}_{2}$ because the spin-orbit coupling in the core level was not taken into account. This spin-orbit splitting is important for L-edges, but not relevant to the K-edges since the $1 s$ level has no spin-splitting. The results for the $\mathrm{P}-\mathrm{L}_{2,3}$-edge are $6.4 \mathrm{eV}$ for the relative peak distances between features $\mathrm{A}$ and $\mathrm{B}, 11.6 \mathrm{eV}$ for $\mathrm{A}$ and $\mathrm{C}, 20.6 \mathrm{eV}$ for $\mathrm{A}$ and $\mathrm{D}$, and $26.7 \mathrm{eV}$ for the features $\mathrm{A}$ and $\mathrm{E}$. There is a major peak at around $156 \mathrm{eV}$ in the calculated ELNES, which appears slightly weaker in the experimental data (D). The smoother peak in the measurement occurring above $164 \mathrm{eV}$ labeled $\mathrm{E}$ is again caused by the $\mathrm{P}-\mathrm{L}_{2}$-edge. The results for the experimental and the calculated peak positions are listed in Table 1 . The difference in the relative peak position is smaller than $1.1 \mathrm{eV}$. Once more, the experimental ELNES is in good agreement with the DFT calculation.

The N-K-edge is given in Figure $3 \mathrm{c}$ revealing that the first maximum in the experimental data appears at $404.6 \pm 0.1 \mathrm{eV}$. This peak is split, and the features are denoted A and B. Minor features are labeled as $\mathrm{C}, \mathrm{D}$, and $\mathrm{E}$. The calculated data is an 

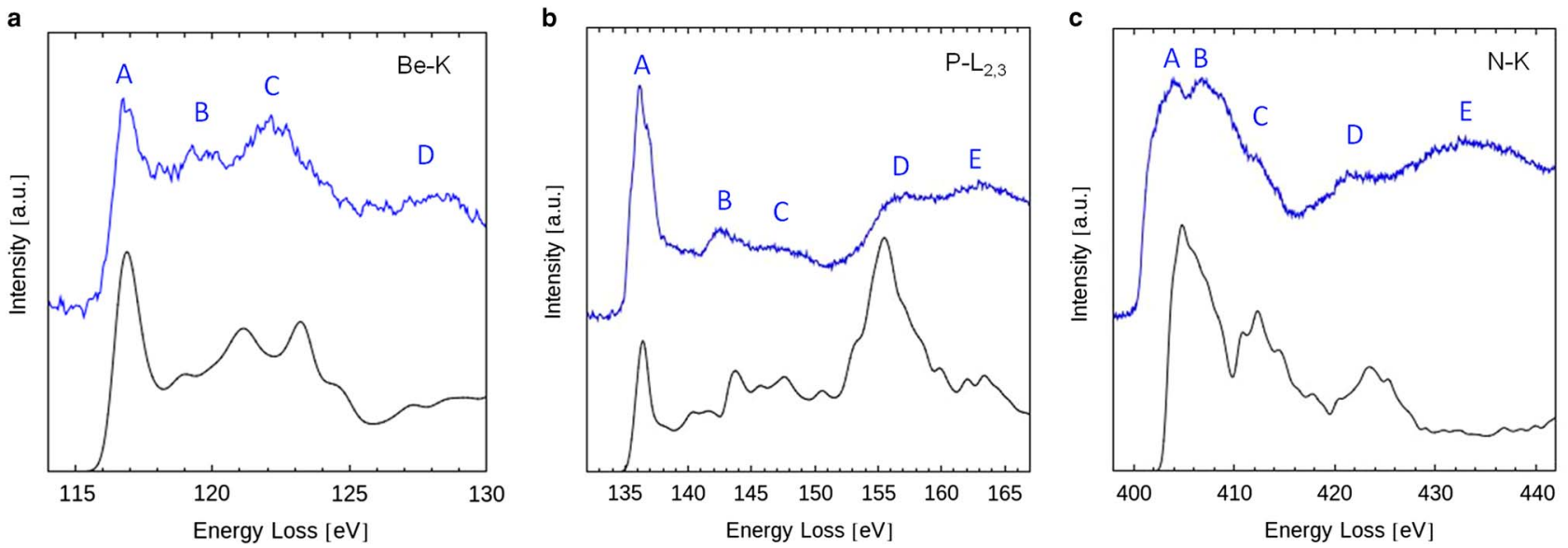

Figure 3. Calculated (Ching et al., 2011) (black) and experimental electron energy-loss spectra (blue) of the phenakitetype $\mathrm{BeP}_{2} \mathrm{~N}_{4}$ showing the Be-K- (a), $\mathrm{P}-\mathrm{L}_{2,3^{-}}(\mathbf{b})$, and $\mathrm{N}$-K-edges $(\mathbf{c})$.

averaged spectrum over the four $\mathrm{N}$ sites (Ching et al., 2011). Here, the first maximum is observed at $407 \mathrm{eV}$. For a better comparison of the relative peak positions the calculated spectrum was shifted by $2.4 \mathrm{eV}$ to lower energy losses. The relative peak distances between features A-E are given in Table 1 . The experimentally measured peak separations are $2.6 \mathrm{eV}$ for features $\mathrm{A}$ and $\mathrm{B}, 8.1 \mathrm{eV}$ for $\mathrm{A}$ and $\mathrm{C}, 17 \mathrm{eV}$ for $\mathrm{A}$ and $\mathrm{D}$, and $29.3 \mathrm{eV}$ for $\mathrm{A}$ and $\mathrm{E}$. The calculated ELNES shows three broader peaks (A, C, and D) with relative peak distances of $7.3 \mathrm{eV}$ for features $\mathrm{A}$ and $\mathrm{C}$ and $18.5 \mathrm{eV}$ for $\mathrm{A}$ and $\mathrm{D}$. The difference between experimental and calculated peak positions of the $\mathrm{N}$-K-edge is between 0.8 and $1.5 \mathrm{eV}$; the experimental and calculated ELNES are in reasonable accordance. The observed double peak labeled A and B in the experimental data is unambiguous, but is not present in the calculated curve where the spectra for the four different $\mathrm{N}$ sites $(\mathrm{N} 1, \mathrm{~N} 2, \mathrm{~N} 3$, and N4 in Fig. 1a) were equally weighted and summed. All four $\mathrm{N}$ atoms have different bond lengths, bond angles, and bond orders leading to different features and peak positions in the $\mathrm{N}$ site-specific ELNES (Ching et al., 2011). The main difference between these four $\mathrm{N}$ sites is that $\mathrm{N} 1$ and $\mathrm{N} 2$ coordinate to each atom type (Be, P1, and P2), while N3 only coordinates to Be and twice to P2 and N4 coordinates to Be and twice to $\mathrm{P} 1$. For the different sites in the relaxed crystal structure, the $\mathrm{N}-\mathrm{P}$ bonds are in all cases shorter than the $\mathrm{N}-\mathrm{Be}$ bonds because the $\mathrm{N}$ valence electrons are pulled closer to the more electronegative $\mathrm{P}$ atoms (compared with $\mathrm{Be}$ ). The total bond order rises in the sequence $\mathrm{N} 1 \quad(1.025)<\mathrm{N} 3 \quad(1.031)<\mathrm{N} 4$ $(1.036)<\mathrm{N} 2(1.039)$. N1 shows the smallest bond order leading to a higher energy loss for the main peak (occurring at position of feature B) whereas N2 has the highest bond order so a lower energy loss for its main peak is observed (occurring at position A). This is in accordance with Brydson et al. (1995) where a longer bond length results in a lower energy of scattering shape resonance and vice versa.

Several reasons might be responsible for the difference of the calculated and experimental N-K-edge. First, using the full core-hole approximation in the DFT calculations (the

Table 1. Position of the First Maxima of the Experimental and Calculated Spectra for the Be-K-, P- $\mathrm{L}_{2,3^{-}}$, and N-K-Edges and their Relative Peak Distances for the Minor Features.

\begin{tabular}{|c|c|c|c|c|c|}
\hline \multirow[b]{2}{*}{ Edges } & \multirow[b]{2}{*}{ First Maximum } & \multicolumn{4}{|c|}{$\begin{array}{l}\text { Relative Peak } \\
\text { Positions }(\mathrm{eV})\end{array}$} \\
\hline & & $\mathrm{A}-\mathrm{B}$ & $\mathrm{A}-\mathrm{C}$ & $\mathrm{A}-\mathrm{D}$ & A-E \\
\hline Experimental Be-K & $116.9 \pm 0.1$ & 2.5 & 5.4 & 11.5 & \\
\hline Calculated Be-K & 118 & 2 & $4.1 / 7.1$ & 12 & \\
\hline$\Delta \mathrm{Be}-\mathrm{K}$ & 1.1 & 0.5 & $1.3 / 1.7$ & 0.5 & \\
\hline Experimental $\mathrm{P}-\mathrm{L}_{2,3}$ & $136.1 \pm 0.7$ & 6.4 & 11.6 & 20.6 & 26.7 \\
\hline Calculated $\mathrm{P}-\mathrm{L}_{3}$ & 138.2 & 7.8 & 11.8 & 19.7 & 27.8 \\
\hline$\Delta \mathrm{P}-\mathrm{L}_{3}$ & 2.1 & 1.4 & 0.2 & 0.9 & 1.1 \\
\hline Experimental N-K & $404.6 \pm 0.1$ & 2.6 & 8.1 & 17 & 29.3 \\
\hline Calculated N-K & $40 \overline{7}$ & & 7.3 & 18.5 & \\
\hline$\Delta \mathrm{N}-\mathrm{K}$ & 2.4 & & 0.8 & 1.5 & \\
\hline
\end{tabular}

The scatter of the peak position is evaluated by averaging over several independent measurements. 
excited electron was placed at the bottom of the $\mathrm{CB}$ ) might not be necessary for the N-K-edge in our compound. In the applied supercell-OLCAO method the excited states are taken into account (Ching et al., 2011). For each edge the ground state of a supercell containing the target atom is calculated first. Then, the final states are calculated separately, where the system remains charge neutral at all times by including the electron in the otherwise empty $\mathrm{CB}$ during charge density accumulation for construction of the self-consistent potential. The CB states exhibit the excited electron from the core (e.g., $1 s$ of Be) placed at the bottom of the $\mathrm{CB}$. The self-consistent iterations of the final-state calculation consider the interaction between the excited electron in the $\mathrm{CB}$ and the hole in the core (Ching et al., 2011). It is known from the literature that, depending on how the core-hole was taken into account, the intensities and peak positions can alter (Lazar et al., 2008). Consequently, another core-hole approximation might describe the splitting of the main peak of the $\mathrm{N}$-K-edge more accurately. This was not studied in the present work since the full core-hole approximation is implemented in the applied code and a change would require additional code development. Second, even a small shift between the individual spectra for the different $\mathrm{N}$ sites can lead to a significant change in the width and position of the peaks appearing in the averaged spectrum. Third, orientation/channeling effects can change the ELNES significantly. We tried to minimize this effect by using out of a low-indexed zone axis orientations. Fourth, the N-K-edge is strongly influenced by point defects such as vacancies. Preliminary inspections of HRTEM images did not give any evidence for a high density of such defects. In any case, the high-energy resolution in the experimental measurement reveals two separate peaks A and $\mathrm{B}$, which can be used to provide guidelines to theoretical schemes for ELNES. A splitting of the N-K-edge has been previously observed in the literature for transition metal nitrides and was related to hybridized nitrogen $2 p$ states and transition metal $3 d$ states with symmetries of $t_{2 g}$ and $e_{g}$ (Paxton et al., 2000; Lazar et al., 2003; Tsujimoto et al., 2005).

Comparing the experimental EELS data to theoretical calculations of the phenakite- and the spinel-type phase (Ching et al., 2011) reveals a tetrahedral coordination for $\mathrm{P}$ and $\mathrm{Be}$ in the phenakite-type structure. A phase transformation under high pressure would lead to a spinel-type, which crystallizes in the face-centered cubic structure with lattice parameters of $a=7.4654 \AA$ (space group $F d \overline{3} m$, no. $227, Z=2$, Ching et al., 2011). In that case, Be atoms would be tetrahedrally coordinated by four $\mathrm{N}$ atoms, whereas the $\mathrm{P}$ atoms would be octahedrally coordinated by six $\mathrm{N}$ atoms. This would lead to a higher coordination number for $\mathrm{P}$ in the spinel-type, from four to six compared with the phenakite-type. A significant change in the ELNES for the $\mathrm{Be}-\mathrm{K}$-, the $\mathrm{P}-\mathrm{L}_{2,3^{-}}$, and the N-K-edge of the spineltype phase has been predicted by Ching et al. (2011). However, experimentally this phase has not yet been successfully synthesized.

Overall, the good agreement between experimental and calculated data reveals that $1 \mathrm{eV}$ broadening in the calculated
EEL spectra is sufficient to describe the experimental broadening occurring due to life time effects of the core-hole and the excited state for our material system. Similar observations, in particular for K-edges (above $400 \mathrm{eV}$ ) have been reported by Kothleitner et al. (2003), Scheu et al. (1998), and Muller et al. (1998). Nevertheless, the use of a monochromator is still beneficial since the individual features in the ELNES are less smeared out due to the reduced tails of the ZLP compared with nonmonochromated data.

\section{ConcLuSIONS}

High-energy-resolution EELS data have been successfully acquired from a new phenakite-type $\mathrm{BeP}_{2} \mathrm{~N}_{4}$ wide-band gap semiconductor without altering the structure. This is important for further development of compounds with ultralight elements since the possibility of their detection is limited. In this study we could not only measure the presence of Be but could also determine its coordination and bonding behavior in the compound. This was demonstrated by a good agreement with calculated ELNES data for the Be-K-, $\mathrm{P}^{-} \mathrm{L}_{3^{-}}$, and (partly) N-K-edges concerning shape, relative peak positions, and edge onsets. To describe the experimental $\mathrm{P}-\mathrm{L}_{2,3}$-edge in more detail, future DFT calculations should include the contributions of the spin-orbit terms. Nevertheless, the experimental measurements confirm that $\mathrm{P}$ and $\mathrm{Be}$ are tetrahedrally coordinated. $\mathrm{N}$ is threefold coordinated and is located on four different sites in the phenakite-type structure. Overall, this type of measurement will allow an easy identification of new structure types such as hypothetical ultrahard spinel-type $\mathrm{BeP}_{2} \mathrm{~N}_{4}$ material in the future.

\section{ACKNOWLEDGMENTS}

The authors acknowledge support of the National Center for Electron Microscopy, Lawrence Berkeley Lab, which is supported by the US Department of Energy under Contract \# DE-AC02-05CH11231, the Deutsche Forschungsgemeinschaft with project SCHN 377/13 as well as the Fonds der Chemischen Industrie. W.Y.C. acknowledges the resources of the National Energy Research Scientific Computing Center supported by the Office of Basic Science of DOE under Contract No. DE-AC03-76SF00098. The authors would like to thank the cluster of excellence Nanosystems Initiative Munich for further financial support.

\section{REFERENCES}

Armenta, M.G.M. \& Reyes-Serrato, A. (2001). Direct wide band gap material: A Hartree-Fock study of $\alpha-\mathrm{Be}_{3} \mathrm{~N}_{2}$. Comput Mater Sci 21, 95-100.

Brydson, R. (2001). Electron Energy Loss Spectroscopy, 1st ed. Oxford: BIOS Scientific Publishers Limited.

Brydson, R., Bruley, J., Müllejans, H., Scheu, C. \& Rühle, M. (1995). Modelling the bonding at metal-ceramic interfaces using PEELS in the STEM. Ultramicroscopy 59, 81-92.

Ching, W.-Y. (1990). Theoretical studies of the electronic properties of ceramic materials. J Am Ceram Soc 73, 3135-3160. 
Ching, W.-Y., Aryal, S., Rulis, P. \& Schnick, W. (2011). Electronic structure and physical properties of the spinel-type phase of $\mathrm{BeP}_{2} \mathrm{~N}_{4}$ from all-electron density functional calculations. Phys Rev B 83, 155109.

Ching, W.-Y. \& Rulis, P. (2009). X-ray absorption near edge structure/electron energy loss near edge structure calculation using the supercell orthogonalized linear combination of atomic orbitals method. J Phys Condens Matter 21, 104202.

CHING, W.-Y. \& Rulis, P. (2012). Electronic Structure Methods for Complex Materials: The Orthogonalized Linear Combination of Atomic Orbitals. Oxford: Oxford University Press.

Egerton, R.F. (2011). Electron Energy-Loss Spectroscopy in the Electron Microscope, 3rd ed. New York: Springer Science+ Business Media.

Erni, R. \& Browning, N.D. (2005). Valence electron energy-loss spectroscopy in monochromated scanning transmission electron microscopy. Ultramicroscopy 104, 176-192.

Gu, L., Srot, V., Sigle, W., Koch, C.T., van Aken, P.A., Scholz, F., Thapa, S.B., Kirchner, C., Jetter, M. \& RÜhle, M. (2007). Bandgap measurements of direct and indirect semiconductors using monochromated electrons. Phys Rev B 75, 195214.

Haider, M., Müller, H., Uhlemann, S., Zach, J., Loebau, U. \& Hoeschen, R. (2008). Prerequisites for a $\mathrm{C}_{\mathrm{c}} / \mathrm{C}_{\mathrm{s}}$-corrected ultrahigh-resolution TEM. Ultramicroscopy 108, 167-178.

Horstmann, S., Irran, E. \& Schnick, W. (1997). Synthesis and crystal structure of phosphorus(V) nitride $\alpha-\mathrm{P}_{3} \mathrm{~N}_{5}$. Angew Chem Int Ed Engl 36, 1873-1875.

KAHL, F. \& Rose, H. (2000). Design of a monochromator for electron sources. In 12th European Congress on Electron Microscopy, Brno, Tománek, P. \& Kolarik R. (Eds.), pp. 1459-1460.

Kothleitner, G., Grogger, W. \& Hofer, F. (2003). Experiencies and possibilities with a $200 \mathrm{kV}$ monochromated (S)TEM. Microsc Microanal 9, 846-847.

Kroll, P. \& Schnick, W. (2002). A density functional study of phosphorus nitride $\mathrm{P}_{3} \mathrm{~N}_{5}$ : Refined geometries, properties, and relative stability of $\alpha-\mathrm{P}_{3} \mathrm{~N}_{5}$ and $\gamma-\mathrm{P}_{3} \mathrm{~N}_{5}$ and a further possible high-pressure phase $\delta-\mathrm{P}_{3} \mathrm{~N}_{5}$ with kyanite-type structure. Chem Eur J 8, 3530-3537.

Kuykendall, T., Ulrich, P., Aloni, S. \& Yang, P. (2007). Complete composition tunability of InGaN nanowires using a combinatorial approach. Nat Mat 6, 951-956.

De la Cruz, W., Soto, G. \& Yubero, F. (2004). Beryllium nitride: An alternative material to beryllium for extreme ultraviolet and soft X-ray uses. Opt Mat 25, 39-42.

Lambrecht, W.R.L. \& Segall, B. (1992). Electronic structure of $\mathrm{BeCN}_{2}$ : A proposed nearly direct wide-band-gap semiconductor. Phys Rev B 45, 1485-1487.

Lazar, P., Redinger, J., Strobl, J., Podloucky, R., Rashkova, B., Dehm, G., Kothleitner, G., Sturm, S., Kutschej, K., Mitterer, C. \& SCHEU, C. (2008). N-K electron energy-loss near-edge structures for TiN/VN layers: An ab initio and experimental study. Anal Bioanal Chem 390, 1447-1453.

LaZar, S., Botton, G.A., Wu, M.-Y., TichelaAr, F.D. \& Zandbergen, H.W. (2003). Materials science applications of HREELS in near edge structure analysis and low-energy loss spectroscopy. Ultramicroscopy 96, 535-546.
Lie, K., Hoier, R. \& Brydson, R. (2000). Theoretical siteand symmetry-resolved density of states and experimental EELS near-edge spectra of $\mathrm{AlB}_{2}$ and $\mathrm{TiB}_{2}$. Phys Rev B 61, 1786-1794.

Liebau, F. (1985). Structural Chemistry of Silicates. Berlin: Springer.

Mo, S.-D. \& CHING, W.-Y. (2000). Ab initio calculation of the corehole effect in the electron energy-loss near-edge structure. Phys Rev B 62, 7901-7907.

Mokhtari, A. \& Akbarzadeh, H. (2003). Ab initio calculations of the electronic and structural properties of beryllium-, magnesium- and calcium-nitrides. Physica B Condens Matter 337, 122-129.

Muller, D., Singh, D. \& Silcox, J. (1998). Connections between the electron-energy-loss spectra, the local electronic structure, and the physical properties of a material: A study of nickel aluminum alloys. Phys Rev B 57, 8181-8202.

Nelhiebel, M., Louf, P.-H., Schattschneider, P., Blaha, P., SchwarZ, K. \& Jouffrey, B. (1999). Theory of orientationsensitive near-edge fine-structure core-level spectroscopy. Phys Rev B 59, 807-814.

Paxton, A.T., van Schilfgatrde, M., MacKenzie, M. \& Craven, A.J. (2000). The near-edge structure in energy-loss spectroscopy: Many-electron and magnetic effects in transition metal nitrides and carbides. J Phys Condens Matter 12, 729-750.

Pucher, F.J., Römer, S.R., Karau, F.W. \& Schnick, W. (2010). Phenakite-type $\mathrm{BeP}_{2} \mathrm{~N}_{4}-\mathrm{A}$ possible precursor for a new hard spinel-type material. Chem Eur J 16, 7208-7214.

Schaffer, B., Grogger, W., Kothleitner, G. \& Hofer, F. (2010). Comparison of EFTEM and STEM EELS plasmon imaging of gold nanoparticles in a monochromated TEM. Ultramicroscopy 110, 1087-1093.

Scheu, C., Dehm, G., Rühle, M. \& Brydson, R. (1998). Electronenergy-loss spectroscopy studies of $\mathrm{Cu}-\alpha-\mathrm{Al}_{2} \mathrm{O}_{3}$ interfaces grown by molecular beam epitaxy. Philos Mag A 78, 439-465.

SCHNick, W., LÜCKe, J. \& KrumeICh, F. (1996). Phosphorus nitride $\mathrm{P}_{3} \mathrm{~N}_{5}$ : Synthesis, spectroscopic, and electron microscopic investigations. Chem Mater 8, 281-286.

Tanaka, I. \& Mizoguchi, T. (2009). First-principles calculations of X-ray absorption near edge structure and energy loss near edge structure: Present and future. J Phys Condens Matter 21, 104201.

Tsujimoto, M., Kurata, H., Nemoto, T., Isoda, S., Terada, S. \& KAJI, K. (2005). Influence of nitrogen vacancies on the $\mathrm{N}$ K-ELNES spectrum of titanium nitride. J Electron Spectrosc Relat Phenomena 143, 159-165.

Uhlemann, S. \& Haider, M. (2002). Experimental set-up of a purely electrostatic monochromator for high resolution and analytical purposes of a $200 \mathrm{kV}$ TEM. Microsc Microanal 8, 584-585.

Zerr, A., Miehe, G., Serghiou, G., Schwarz, M., Kroke, E., Riedel, R., Fueß, H., Kroll, P. \& Boehler, R. (1999). Synthesis of cubic silicon nitride. Nature 400, 340-342.

Ziegler, A., Idrobo, J.C., Cinibulk, M.K., Kisielowski, C., Browning, N.D. \& Ritchie, R.O. (2004). Interface structure and atomic bonding characteristics in silicon nitride ceramics. Science 306, 1768-1770. 